\title{
TEAM BASED LEARNING: UMA METODOLOGIA ATIVA PARA AUXILIO NO PROCESSO DE APRENDIZAGEM
}

Ana Paula Ambrósio Zanelato Marques, Carla Plantier Message, Raquel Rosan Cristino Gitahy, Adriana Lima Terçariol.

Universidade do Oeste Paulista - UNOESTE, Mestrado em Educação, Presidente Prudente, SP. E-mail: anapaulazanelato@gmail.com.

\section{RESUMO}

Os métodos de aprendizagem tradicionais, consistidos em atividades de ouvir e falar, são incapazes de motivar e desenvolver características necessárias nos estudantes. Os métodos de aprendizagem ativos são focados no aluno, portanto, mais condizentes com esta geração, os chamados "nativos digitais". Este artigo possui como foco o método ativo de Aprendizagem Baseada em Equipes ou Team Based Learning (TBL). Apresentaremos os conceitos e as etapas de aplicação do método. Para este estudo, realizamos um estudo bibliográfico em bases de dados como Portal da Capes, Scielo, IBICT e Banco de testes e dissertações da USP. Constatamos que existe pouca publicação sobre o tema em português. Concluímos que o TBL promove o trabalho colaborativo, a autonomia e proatividade dos estudantes, buscando melhorar a aprendizagem por meio de uma inversão da sala de aula, focado na comunicação, responsabilidade entre os pares e a auto-aprendizagem.

Palavras-Chave: Aprendizagem Baseada em Equipes, Metodologia ativa, Método Colaborativo.

\section{TEAM BASED LEARNING: AN ACTIVE TEACHING METHODOLOGY FOR ASSISTANCE IN THE LEARNING PROCESS}

\begin{abstract}
Traditional learning methods, consisting of listening and speaking activities, are incapable of motivating and developing characteristics necessary the students. The active teaching methods are focused on the student, therefore, more in keeping with this generation, of so-called "digital natives". This article focuses on the active method of Team Based Learning (TBL). We will present the concepts and the steps of application of the method. For this study, we carried out a bibliographic study in databases such as Portal da Capes, Scielo, IBICT and Bank of tests and dissertations of USP. We found that there is little publication on the subject in Portuguese. We conclude that TBL promotes collaborative work, autonomy and proactivity of students, seeking to improve learning through a classroom inversion, focused on communication, between peer responsibility and self-learning.
\end{abstract}

Keywords: Team Based Learning. Active Methodology, Collaborative method. 


\section{INTRODUÇÃO}

Atualmente, milhares de informações novas são disponibilizadas diariamente, devido ao advento da tecnologia, cada vez mais presente em nosso ambiente, obter informações sobre qualquer assunto em qualquer lugar tornou-se extremamente acessível e fácil, essa é a realidade em que muitos jovens já nasceram e cresceram, sendo esta é a geração presente nas salas de aulas.

Diante deste cenário, os métodos de aprendizagem tradicionais, que consistem em atividades de ouvir e falar, obtêm pouco êxito nestes estudantes, uma vez que já estão completamente imergidos neste mundo digital, os professores já não são mais os detentores do conhecimento.

Para Savegnago (2015, p.15), “Quando estudantes são ensinados por métodos tradicionais passivos de ensino, existe pobre transferência de informação, entendimento e retenção de conhecimento".Segundo Oliveira (2016, p.11), "Atrelada a essa concepção de ensino está a desmotivação do discente, caracterizada por alunos cada vez menos engajados no processo de aprendizagem e que desistem diante dos desafios menores, por não acreditarem em suas próprias capacidades"

William Glasser (1925-2013), um psiquiatra americano explica em sua pesquisa "Como Aprendemos"1 ${ }^{1}$ grau de aprendizagem de acordo com a técnica utilizada, para isso ele criou a Pirâmide da Aprendizagem (Figura 1). Quando o aluno apenas escuta o conteúdo, como no caso das aulas expositivas, ele consegue reter apenas $20 \%$, por outro lado, uma aprendizagem mais ativa, como quando o aluno realiza a prática, este índice aumenta para $80 \%$, ou seja, uma aprendizagem mais significativa é aquela na qual o aluno torna-se o centro do processo, onde o processo de aprendizagem é mais colaborativo e motivador.

Figura 1. Pirâmide de Aprendizagem de William Glasser

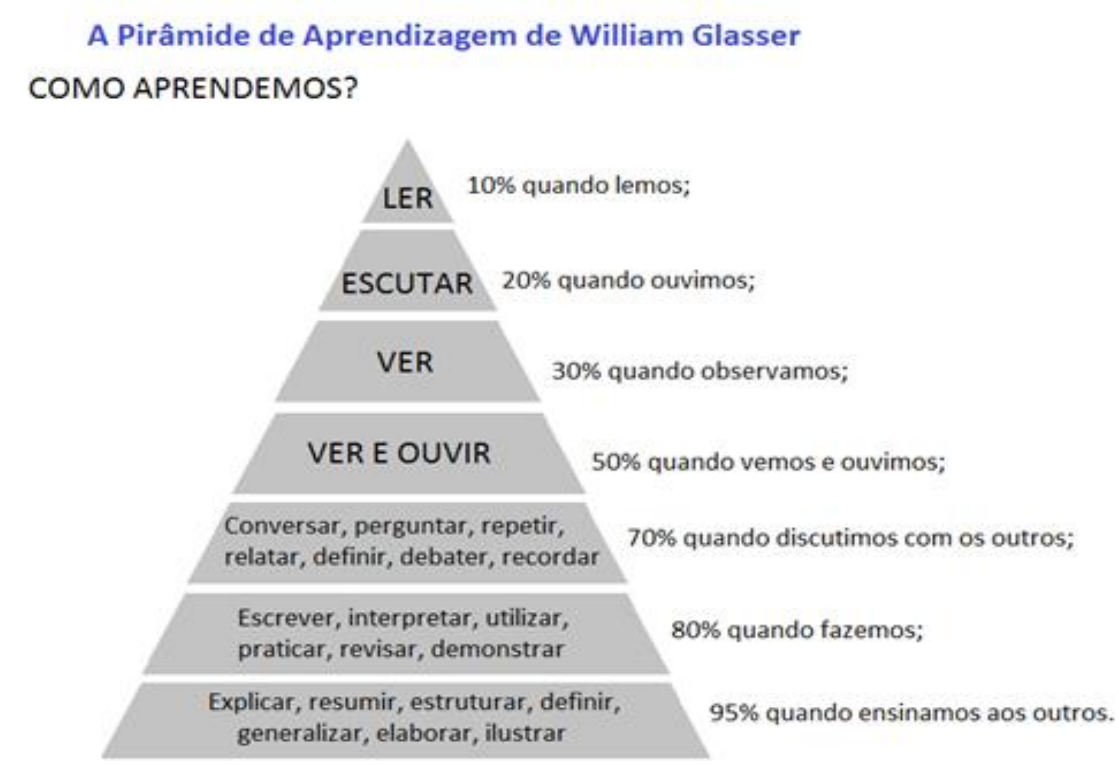

Fonte: Adaptada pelos autores da Pirâmide de Aprendizagem de William Glasser

Os docentes necessitam rever suas práticas pedagógicas, buscando meios alternativos que consigam tornar o processo de aprendizagem mais colaborativo e motivador, envolvendo mais os estudantes para que se tornem protagonistas do seu conhecimento. A nova geração, os chamados "nativos digitais" possuem informação disponível a todo o momento, entretanto, ainda precisam de ajuda para tornar estas informações fontes de conhecimento. Neste contexto, quais estratégias

\footnotetext{
${ }^{1}$ Como Aprendemos? Disponível em <http://ochoamores.typepad.com/morespanish/2013/07/c\%C3\%B3moaprendemos.html\#comment-6a00e552126133883301b8d0d03017970c>. Acesso em 25 jul. 2017.
} 
de aprendizagem podemos buscar para conseguir atingir tais objetivos? Os métodos ativos podem ser uma alternativa viável, que possibilita desenvolvermos atitudes mais ativas dos estudantes, exercitando a criatividade, o trabalho colaborativo, tornando-se tomadores de decisões, com atitudes proativas, sendo agentes de transformação.

Os métodos tradicionais, como aulas expositivas apenas transfere a informação do docente para o aluno, já os métodos educacionais ativos, "[...] consistem de elementos de falar, ouvir, escrever, ler e refletir, recrutam uma variedade de funções cerebrais e capacitam os estudantes a criar estruturas mentais mais significativas, transferíveis e duráveis" (FIALHO, 2015, p.16).

Conforme descrito por Dias (2016, p.02), as metodologias ativas possuem as seguintes características:

- Centradas no aluno;

- Envolvem métodos e técnicas que estimulam a interação aluno $x$ professor, aluno $x$ aluno e aluno $x$ material didático e outros recursos de aprendizagem.

- Opõem-se a métodos e técnicas que enfatizam a transmissão do conhecimento.

- Têm como bases teóricas:

- Sócio construtivismo;

- Psicologia cognitiva;

- Teoria cognitiva social;

- Aprendizagem baseada na reflexão;

- Aprendizagem colaborativa e significativa;

- Reflexão crítica sobre a experiência;

- Maior apropriação e divisão das responsabilidades no processo de ensino-aprendizagem;

- Desenvolvimento de capacidade para auto-aprendizagem;

- Favorece uma maior retenção do conhecimento;

- Produz melhoria no relacionamento interpessoal.

Segundo Vendrame e Vendrame (2014), existem várias metodologias de aprendizagem ativas, dentre elas podemos citar: Peer Instruction (Aprendizagem pelos Pares), Project Based Learning (Aprendizagem Baseada em Projetos), Problem Based Learning (Aprendizagem Baseada em Problemas), Flip Class (Classe Invertida), Aprendizagem Hibrida, entre outras. Neste trabalho, focaremos no método de aprendizagem ativo Team Based Learning (TBL), ou Aprendizagem Baseada em Equipes, possuindo por objetivos a melhora da aprendizagem por meio do trabalho colaborativo.

Este artigo apresenta o método ativo TBL, seus conceitos e características, exemplificando o processo de aplicação e seus benefícios na aprendizagem. Para o seu desenvolvimento, fez-se um estudo exploratório, a partir de um levantamento bibliográfico nas bases de dados do Scielo, do Portal da Capes, Banco de testes e dissertações da USP e plataforma IBICT.

\section{METODOLOGIA}

Para este estudo realizou-se uma revisão de literatura, nesta pesquisa, realizamos uma busca por artigos científicos, filtrando pelo período de 2008 a 2017 nas bases de dados do Scielo, Portal da Capes, Banco de testes e dissertações da USP e plataforma IBICT. Todas as bases foram selecionadas pelo fato de disponibilizarem a busca online em artigos científicos em periódicos bem avaliados. Os descritores utilizados para a busca inicial foram: "Aprendizagem Baseada em 
Equipes" e o mesmo termo em inglês, "Team Based Learning". Na sequência, foram realizadas leituras de todos os resumos dos artigos disponibilizados afim de verificar se eles estavam relacionados com os objetivos deste artigo, exceto os artigos do portal da Capes, que devido ao grande número de artigos encontrados em inglês e muitos deles estarem indisponíveis no Portal, muitos foram desconsiderados. Os artigos selecionados foram completamente lidos e fichados. $\mathrm{O}$ quadro a seguir apresenta informações sobre os artigos encontrados e os selecionados.

Quadro 1. Artigos encontrados e selecionados

\begin{tabular}{|c|c|c|c|c|c|c|c|c|c|c|c|c|}
\hline & \multicolumn{4}{|c|}{ Total de artigos } & \multicolumn{4}{|c|}{ Publicados em língua } & \multicolumn{4}{|c|}{ Artigos selecionados } \\
\hline Descritores & $\begin{array}{c}\text { Sciel } \\
0\end{array}$ & $\begin{array}{c}\text { Cap } \\
\text { es }\end{array}$ & $\begin{array}{c}\text { IBIC } \\
T\end{array}$ & $\begin{array}{l}\text { Test } \\
\text { es e } \\
\text { Diss. }\end{array}$ & $\begin{array}{c}\text { Sciel } \\
0\end{array}$ & $\begin{array}{c}\text { Cape } \\
\mathbf{S}\end{array}$ & $\begin{array}{c}\text { IBIC } \\
\mathbf{T}\end{array}$ & $\begin{array}{l}\text { Test } \\
\text { es e } \\
\text { Diss. }\end{array}$ & $\begin{array}{c}\text { Sciel } \\
0\end{array}$ & $\begin{array}{c}\text { Cap } \\
\text { es }\end{array}$ & $\begin{array}{c}\text { IBIC } \\
\text { T }\end{array}$ & $\begin{array}{l}\text { Test } \\
\text { es e } \\
\text { Diss. }\end{array}$ \\
\hline $\begin{array}{l}\text { "Aprendizag } \\
\text { em Baseada } \\
\text { em Equipes" }\end{array}$ & 1 & 2 & 4 & 7 & 2 & $\begin{array}{c}2 \\
\text { Port. }\end{array}$ & $\begin{array}{c}4 \\
\text { Port } \\
. \\
\end{array}$ & $\begin{array}{c}7 \\
\text { Port. }\end{array}$ & 1 & 1 & 2 & 1 \\
\hline $\begin{array}{c}\text { "Team } \\
\text { Based } \\
\text { Learning" }\end{array}$ & 7 & 1566 & 0 & 0 & $\begin{array}{c}1 \\
\text { Ing., } \\
4 \\
\text { Esp. } \\
2 \\
\text { Port. }\end{array}$ & $\begin{array}{c}1150 \\
\text { Ing., } \\
10 \\
\text { Esp. , } \\
1 \\
\text { Port } \\
\text { e } 38 \\
\text { outro } \\
\text { s. }\end{array}$ & 0 & 0 & 1 & 1 & 0 & 0 \\
\hline
\end{tabular}

Fonte: Pesquisa Bibliográfica, 2017.

Diante da busca realizada encontra-se muito material publicado no exterior, em especial em inglês, entretanto, na busca pelo termo em português a quantidade de artigos encontrados se reduz significativamente. Concluindo-se que existem poucos materiais publicados em português sobre este termo, tornando uma justificativa o estudo e publicação do método TBL no Brasil. No quadro abaixo, apresentamos os artigos selecionados, totalizando 6 artigos ${ }^{2}$, pois, muitos eram apareciam em mais de uma busca pelos termos, repetindo-se.

\footnotetext{
${ }^{2}$ Com o intuito de garantir mais consistência neste trabalho, acrescentamos outras leituras com os mesmos objetivos para criação deste artigo, procedentes de livros, capítulos de livros, dissertação de mestrado e trabalhos publicados em anais.
} 
Quadro 2. Artigos selecionados nas bases do Scielo e do Portal da Capes

\begin{tabular}{|c|c|c|c|}
\hline Artigo & Revista & $\begin{array}{l}\text { Publica } \\
\text { ção }\end{array}$ & Base \\
\hline $\begin{array}{l}\text { O “Bê-Á-Bá" da Aprendizagem Baseada em Equipe. Rodrigo de Rosso } \\
\text { Krug et al. }\end{array}$ & Rev. bras. educ. med. & 2016 & Scielo \\
\hline TBL - Aprendizaje Basado en Equipos. Daniel Moraga, a Jeannette Soto & $\begin{array}{l}\text { Estudios pedagógicos } \\
\text { (Valdivia) }\end{array}$ & 2016 & Scielo \\
\hline $\begin{array}{l}\text { A construção do trabalho de conclusão do curso por meio da } \\
\text { metodologia ativa team-based learning. Ani Mari Hartz, Gabriel Vianna } \\
\text { Schlatter }\end{array}$ & RAEP & 2016 & Portal Capes \\
\hline $\begin{array}{l}\text { Aprendizagem baseada em equipe: uma estratégia de ensino aplicada } \\
\text { na área da imaginologia. Rosana Maria Paiva dos Anjos et al. }\end{array}$ & $\begin{array}{l}\text { Revista da Faculdade } \\
\text { de Ciências Médicas } \\
\text { de Sorocaba }\end{array}$ & 2016 & Portal Capes \\
\hline $\begin{array}{l}\text { Metodologias ativas peer instruction e team based learning na } \\
\text { formação inicial de professores de matemática. Greyson Alberto Rech }\end{array}$ & Plataforma Sucupira & 2016 & $\begin{array}{l}\text { Banco de } \\
\text { teste e } \\
\text { Dissertações } \\
\text { USP } \\
\end{array}$ \\
\hline $\begin{array}{c}\text { Avaliação de proposta estruturada na aprendizagem baseada em } \\
\text { equipes (tbl team-based learning) para utilização em um curso de } \\
\text { medicina. Carlos Alberto de Oliveira, Maria Helena Senger }\end{array}$ & $\begin{array}{l}\text { Dissertação da } \\
\text { Pontifícia Universidade } \\
\text { Católica de São Paulo }\end{array}$ & 2014 & $\mathrm{IBICT}$ \\
\hline $\begin{array}{c}\text { Avaliação do homem virtual em hanseníase na aprendizagem baseada } \\
\text { em equipes (team-based learning) na graduação médica. Cristiane } \\
\text { ComparinSavegnago, GünterHans Filho }\end{array}$ & Dissertação UFMS & 2015 & $\mathrm{IBICT}$ \\
\hline $\begin{array}{l}\text { Team-Based Learning in US Colleges and Schools of Pharmacy. } \\
\text { ALLEN, R. E., et al. }\end{array}$ & $\begin{array}{l}\text { American Journal of } \\
\text { Pharmaceutical } \\
\text { Education }\end{array}$ & 2013 & Capes \\
\hline
\end{tabular}

Fonte: Pesquisa Bibliográfica, 2017.

\section{DISCUSSÃO}

O Team Based Learning (TBL) ou Aprendizagem Baseada em Equipes é um método ativo, que envolve o trabalho colaborativo, possuindo como foco o estudante, promovendo a autonomia e proatividade.

O Team-Based Learning, foi criado pelo professor de gestão e negócios Larry Michaelsen, no final dos anos 70, na universidade de Oklahoma (EUA). O método tem como foco melhorar a aprendizagem e desenvolver habilidades de trabalho colaborativo, através de uma estrutura que envolve: o gerenciamento de equipes de aprendizagem, tarefas de preparação e aplicação de conceitos, feedback constante e avaliação entre os colegas. A ideia central é que os alunos se sintam responsáveis pela própria aprendizagem e pela dos colegas (MICHAELSEN, KNIGHT; FINK, 2004).

O TBL busca melhorar a aprendizagem realizando a inversão da sala de aula, focado na comunicação, responsabilidade entre os pares e autoaprendizagem. O processo de aplicação do TBL ocorre em fases. A figura 2 abaixo exemplifica as etapas. 
Figura 2. Etapas de Aplicação do TBL

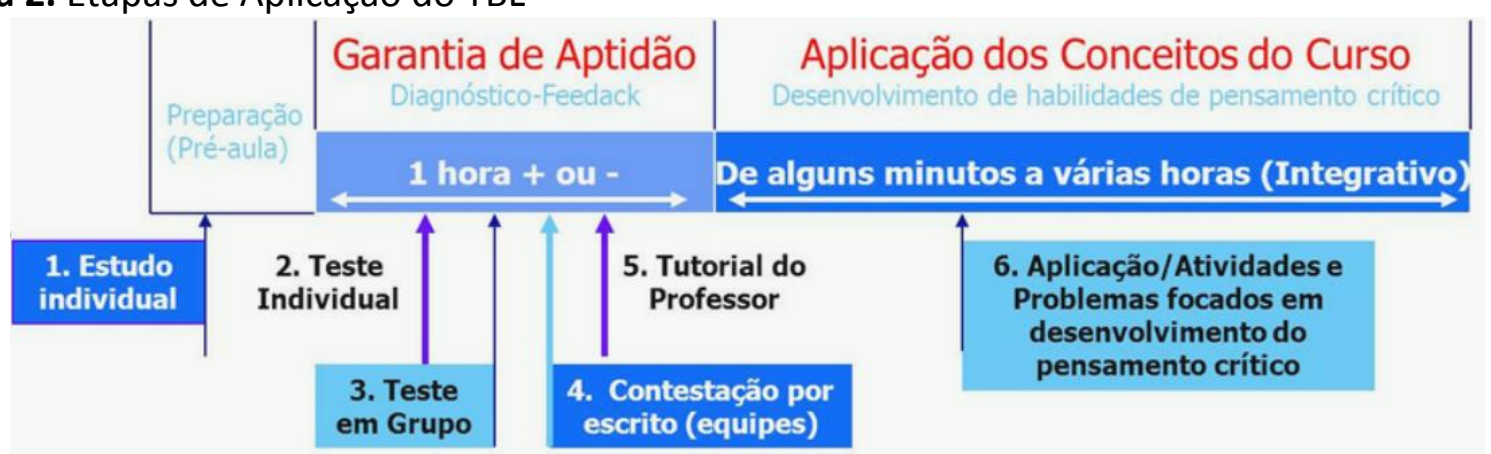

Fonte: adaptada pelos autores de Michaelsen, Sweet e Parmelee (2008, p. 05)

Na implementação do TBL as fases são:

- $\quad$ Fase da preparação: o docente disponibiliza materiais de estudo aos alunos querealizam um estudo prévio antes da aula.

- $\quad$ Teste de preparação individual (Figura 3): em sala de aula, os estudantes respondem um teste conceitual individual, preenchendo um gabarito.

- $\quad$ Teste de preparação em equipe (Figura 4): Os estudantes discutem em equipes, e respondem o mesmo teste em um gabarito de feedback imediato, espécie de "raspadinha". Em caso de erro, os estudantes voltam a discutir, para encontrar a resposta correta.

- Contestação por escrito: nesta etapa os estudantes podem realizar apelações de questões que possam ter respostas ambíguas ou que estejam mal formuladas.

- Exposição Oral: O professor finaliza a fase de preparação realizando uma pequenaexposição oral.

- Tarefas de aplicação: são atividades realizadas individualmente ou em equipes, geralmente do tipo resolução de problemas.

Figura 3. Teste de Garantia de Preparo Individual

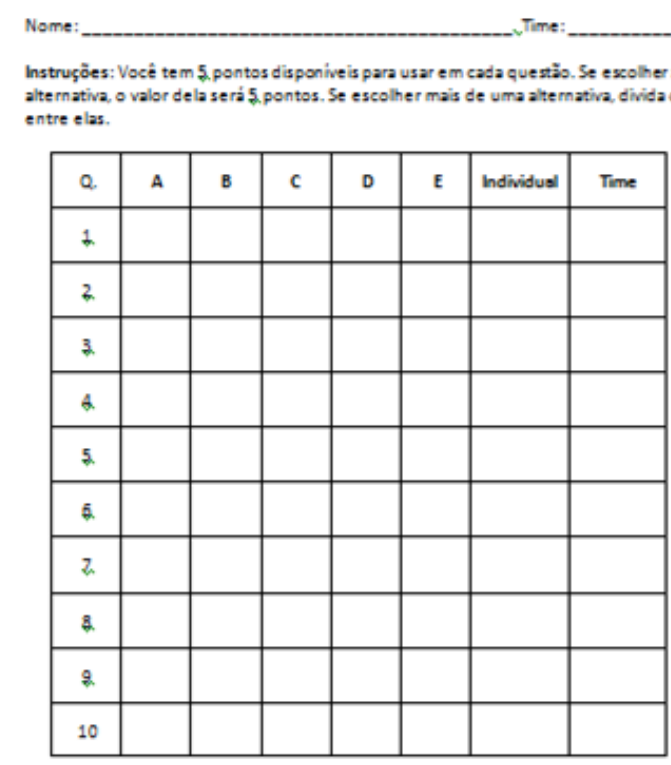

Fonte: Criada pelos autores 
Figura 4. Teste de Garantia de Preparo em Equipes

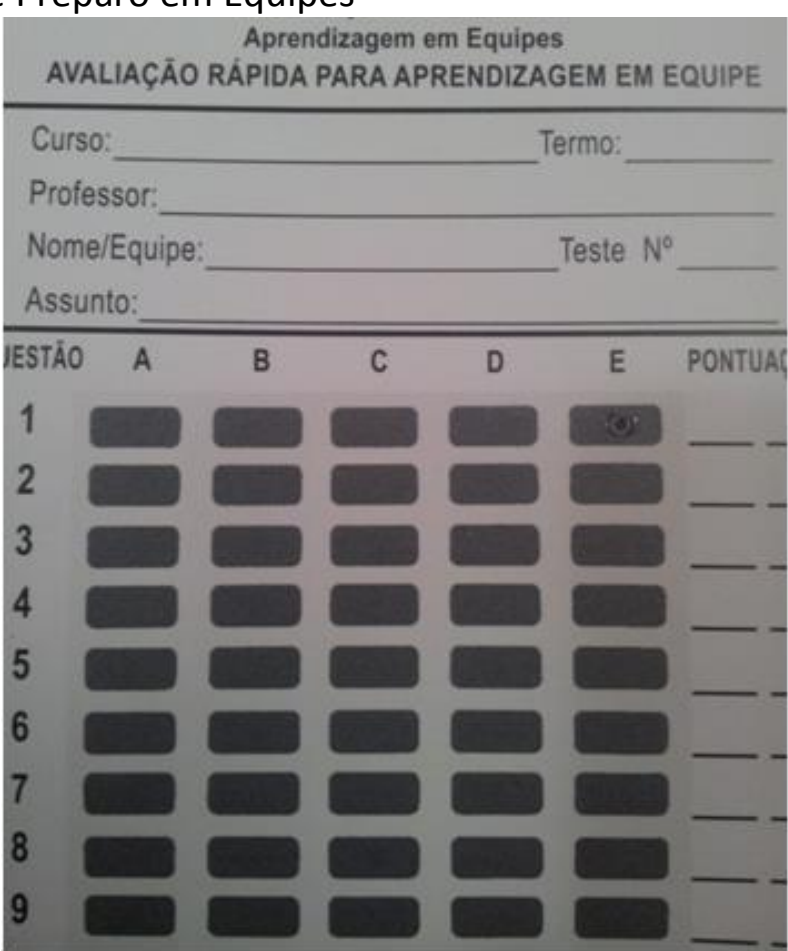

Fonte: Imagem registrada pelos autores

\section{CONCLUSÃO}

Iniciamos este artigo discutindo as necessidades de novas praticas pedagógicas ao aprendizagem, que possam focar no aluno e motivá-lo na busca pelo conhecimento. Apresentamos o método ativo TBL, seus conceitos e passos de aplicação. O TBL tem se mostrado uma forma colaborativa de promover a aprendizagem, mais eficaz e que promove o trabalho em equipe, além da auto-aprendizagem.

Existem inúmeras vantagens que se manifestam quando utilizamos o TBL, como:

- O estudante se habitua a buscar pelo conhecimento devido aos estudos prévios, buscando por outras fontes de conhecimento e facilitando as discussões em sala;

- Devido aos estudos prévios, o estudante passa a ter maior domínio e compreensão do tema estudado, aumentando sua participação em sala;

- Desenvolve habilidades de comunicação entre os pares, na discussão em equipes, ao defender suas escolhas se expondo entre os colegas;

- A aprendizagem ativa ocorre em todas as etapas do TBL, sendo o estudante o foco da metodologia o tempo todo;

- Os estudantes se divertem mais, com isso a aprendizagem de torna mais motivadora, garantindo uma maior eficácia e compreensão.

Observamos que apesar das vantagens relatadas sobre o uso desta metodologia de aprendizagem ativo, a pesquisa revelou que existem poucos artigos em Português sobre o tema, sendo importante mais estudos para divulgação do método TBL.

\section{REFERÊNCIAS}

ALLEN, R. E., et al.Team-Based Learning in US Colleges and Schools of Pharmacy. 2013. American Journal of Pharmaceutical Education, 77(6), 115. Disponível em: http://doi.org/10.5688/ajpe776115. Acesso em: 14 jul. 2017, https://doi.org/10.5688/ajpe776115. ANJOS, R. M. P. et al.. Aprendizagem baseada em equipe: uma estratégia de ensino aplicada na área da imaginologia. 2016.2 Disponível em: <https://doaj.org/article/534db496b0fd43aca423c9deabf435e7 >. Acesso em: 14 jul. 2017. 
CARVALHO, Fatima Franco; CHING, Hong Yuh(Org.). Práticas de ensino Aprendizagem no ensino superior.Rio de Janeiro: Alta Books, 2016. 288 p.

DEWEY, J. Democracia e Educação: introdução à filosofia da educação. 3. ed. São Paulo:

Companhia Editora Nacional, 1959.

DIAS, M.M. Metodologias Ativas: Parte 1. Disponivel em

<http://ned.unifenas.br/blogtecnologiaeducacao/educacao/metodologias-ativas-parte-1/>.

Acesso em: 11 dez. 2016.

FIALHO, Neusa Nogueira. Os Jogos Pedagógicos Como Ferramentas de Ensino. Anais do VIII Congresso Nacional de Educação - Educere. [recurso eletrônico] Curitiba: Champagnat, 2008. Disponível em:

<http://www.pucpr.br/eventos/educere/educere2008/anais/pdf/293_114.pdf>. Acesso em: 27 jul. 2015.

FREIRE, Paulo. Pedagogia da Autonomia: saberes necessários à prática educativa. São Paulo: Paz e Terra, 2011a.

KRUG, Rodrigo de Rosso et al. O “Bê-Á-Bá" da Aprendizagem Baseada em Equipe. 2016. Disponível em: <http://www.scielo.br/scielo.php?script=sci_arttext\&pid=S010055022016000400602\&lang=pt>. Acesso em: 14 jul. 2017

HARTZ, A. M.; SCHLATTER, G, V.. A construção do trabalho de conclusão do curso por meio da metodologia ativa team-based learning. 2016. Disponível em: <https://raep.emnuvens.com.br/raep/article/view/274 >. Acesso em: 14 jul. 2017

MASETTO. M. T. Mediação pedagógica e o uso da tecnologia. In MORAN, J. M.;

MASETTO, M. T.; BEHRENS, M. A. Novas tecnologias e mediação pedagógica. Campinas: Papirus, 2000. P. 139

MICHAELSEN, Larry K.; SWEET, Michael; PARMELEE, Dean X.. Team-Based Learning: Small-Group Learning's Next Big Step. San Francisco: Wiley, 2008.

MICHAELSEN, L.K.; KNIGHT, A.B.; FINK, L.D. Team-Based Learning: A Transformative Use of Small Groups in College Teaching. Sterling, VA: StylusPublishing, LLC, 2004.

MORAGA, D.; SOTO, J. TBL - Aprendizaje Basado en Equipos. 2016. Disponível em:

<http://www.scielo.cl/scielo.php?script=sci_arttext\&pid=S0718-07052016000200025\&lang=pt>. Acesso em: 14 jul. 2017.

MORAN, J. Mudando a Educação com Metodologias Ativas. Disponível em

<http://www2.eca.usp.br/moran/wp-content/uploads/2013/12/mudando_moran.pdf>. Acesso em: 11 dez. 2016.

MORAN, J.M. Metodologias Inovadoras com Tecnologias. Entrevista a João Matar. Disponível em: <https://www.youtube.com/watch?v=pKi2K_xcTGM\&feature=youtu.be>. Acesso em: 13 Jan. 2017. 
OLIVEIRA, Carlos A. de. Metodologia ativa de ensino-aprendizagem: Manual do TBL. E-book: Edição do Autor, 2015.

OLIVEIRA, Tobias Espinosa de. Aprendizagem de física, trabalho colaborativo e crenças de autoeficácia: um estudo de caso com o método Team-Based Learning em uma disciplina introdutória de eletromagnetismo. 2016. Disponível em: <http://www.lume.ufrgs.br/handle/10183/135013>. Acesso em: 28 jun. 2016.

OLIVEIRA, C. A. de; SENGER, M. H. Avaliação de proposta estruturada na aprendizagem baseada em equipes (tbl team-based learning) para utilização em um curso de medicina. 2014. Disponível

em: http://bdtd.ibict.br/vufind/Record/PSP_07c6f28f30fbacad217afe681976463f>Acesso em: 14 jul. 2017

SAVEGNAGO, Cristiane Comparin. Avaliação do Homem Virtual em Hanseníase na aprendizagem baseada em equipes (Team-Based Learning) na Graduação Médica. 2015. Disponível em: <http://repositorio.cbc.ufms.br:8080/jspui/handle/123456789/2642>. Acesso em: 28 jun. 2016. SILBERMAN, M. Active learning: 101 strategies do teach any subject. Massachusetts: Allynand Bacon, 1996.

VENDRAME, F.C; VENDRAME, M.C.R. As Metodologias Ativas de Aprendizagem. Anais do 25은 ENANGRD. Belo Horizonte, MG: 2014.

RECH, G. A. Metodologias ativas peer instruction e team based learning na formação inicial de professores de matemática. 2016.2 Disponível em: https://sucupira.capes.gov.br/sucupira/public/consultas/coleta/trabalhoConclusao/viewTrabalho Conclusao.jsf?popup=true\&id_trabalho=4873792>Acesso em: 14 jul. 2017 